\title{
Nitric oxide alterations in cardiovascular system of rats with Parkinsonism induced by 6-OHDA and submitted to previous exercise
}

\author{
Lorena de Jager ${ }^{\mathrm{a}}$, Eric Diego Turossi Amorim ${ }^{\mathrm{a}}$, Bruno Fernando Cruz Lucchetti ${ }^{\mathrm{a}}$, \\ Fernanda Novi Cortegoso Lopes $^{\mathrm{a}}$, Carlos Cesar Crestani ${ }^{\mathrm{b}}$, Phileno Pinge-Filho ${ }^{\mathrm{c}}$, \\ Marli Cardoso Martins-Pinge ${ }^{a, *}$

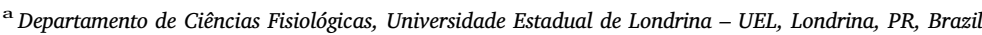 \\ ${ }^{\mathrm{b}}$ Faculdade de Ciências Farmacêuticas de Araraquara, Departamento de Princípios Ativos Naturais e Toxicologia, Universidade Estadual Paulista - UNESP, Araraquara, \\ Brazil \\ ${ }^{\mathrm{c}}$ Departamento de Ciências Patológicas, Universidade Estadual de Londrina - UEL, Londrina, PR, Brazil
}

\section{A R T I C L E I N F O}

\section{Keywords:}

Arterial pressure

L-NAME

Heart rate

Swimming

Parkinson's disease

\begin{abstract}
A B S T R A C T
Studies showed that physical exercise decreases the risk of developing Parkinson's disease (PD) as slowing its progression. Nitric oxide (NO) increases in the substantia nigra pars compacta (SNpc) of individuals with PD. However, no study has evaluated the effects of exercise on peripheral NO levels and its modulatory effects on cardiovascular dysfunctions of subjects with PD. Trained (T) or sedentary (S) animals underwent stereotactic surgery for bilateral 6-hydroxydopamine (6-OHDA) or vehicle microinfusion (Sham group). After 6 days, the animals were catheterized for baseline parameters, followed by inhibition of NOS by Nw-nitro-arginine-methyl ester (L-NAME, $10 \mathrm{mg} / \mathrm{kg}$ - i.v.). Nitrite concentration was performed in the aorta, heart, kidney, adrenal and plasma. After exercise, the animals presented resting bradycardia (6-OHDA T and Sham T). NO was increased in the aorta of 6-OHDA S, and decreased in 6-OHDA T animals. In the heart, NO was increased in Sham T compared to sedentary and decreased in 6-OHDA T relative to 6-OHDA S and Sham T animals. At the kidney, NO decrease in 6-OHDA $S$ and Sham $T$ when compared to Sham $S$ and, in adrenal gland, there was a decrease in 6-OHDA T in relation to 6-OHDA S. L-NAME promoted lower increases in MAP in 6-OHDA groups. The decreases of HR were enhanced due to physical training. 6-OHDA S group presented decreased systolic arterial pressure variability, not altered by exercise. Our data showed alterations in peripheral NO in the association of exercise with Parkinsonism in the cardiovascular function.
\end{abstract}

\section{Introduction}

Parkinson's disease (PD) is characterized by loss of dopaminergic neurons in the substantia nigra pars compacta (SNpc), responsible for most disease-related motor dysfunctions [1,2]. However, patients also present dysautonomia that compromise their quality of life [3]. Among these are: postural and postprandial hypotension, blood pressure (BP) lability, fatigue and intolerance to exercise [4]. Previous studies in animals with bilateral 6-hydroxydopamine (6-OHDA) injury, showed a decrease in mean arterial pressure (MAP) and heart rate (HR) parameters, accompanied by reduced modulation of systolic blood pressure ([5]). In addition, responses to baroreflex and chemoreflex are altered in these animals $[6,7]$. Clinical studies observed that PD patients have lower heart rate variability (HRV) and baroreflex sensitivity (BRS) [8].

Regular exercise promotes several beneficial effects, such as: increased mitochondrial energy production, stimulating antioxidant defenses, reducing inflammation, resting bradycardia, angiogenesis and synaptogenesis, improved baroreflex gain and sensitivity, and autonomic modulation ([9-13]. Animals that exercise before (12 weeks) and after ( 8 weeks) the 6-OHDA injury have a lower decrease in tyrosine hydroxylase (TH) [13]. Mice that underwent a swimming protocol for 4 weeks before exposure to 6-OHDA presented lower generation of reactive oxygen species (ROS), reduction of levels of IL-1 $\beta$, reduced production of glutathione reductase (GR) and glutathione-S-transferase (GST) [14]. Also, physical exercise for 4 weeks promotes angiogenesis in the brain of mice with chronic Parkinsonism [15]. Thus, physical exercise could work as a non-pharmacological therapy to patients with PD.

Nitric oxide (NO) is involved in processes leading to neurodegeneration in Parkinson's disease [16,17]. Administration of NOS

\footnotetext{
* Corresponding author at: Departamento de Ciências Fisiológicas, Centro de Ciências Biológicas, Universidade Estadual de Londrina, Rodovia Celso Garcia Cid, Km 380, Campus Universitário, CEP 86055-900 Londrina, PR, Brazil.

E-mail address: martinspinge@uel.br (M.C. Martins-Pinge).
} 
inhibitors, such as Nw-nitro-arginine-methyl ester (L-NAME) attenuates the decrease of dopamine (DA) by unilateral injection of 6-OHDA [50]. Furthermore, inhibition of nNOS protects against damage of dopaminergic neurons and decreases motor behavior and dyskinesia induced by L-DOPA in parkinsonian rodents [18]. The NOS isoforms appear to behave differently in PD. In the case of nNOS, the degeneration of dopaminergic neurons leads to a decrease in nNOS concentrations in the substantia nigra [19], whereas the administration of MPTP in mice leads to a significant elevation of iNOS [20]. In humans, increased iNOS expression in SNpc was found in brain postmortem samples from individuals with PD [21].

Considering that physical exercise benefits PD patients, and studies that relate parkinsonism with cardiovascular dysfunctions did not evaluate NO participation in the cardiovascular system, the goal of this study was to evaluate the effects of previous physical training in cardiovascular dysfunction, NO concentrations in the cardiovascular system of rats induced to parkinsonism by bilateral infusion of 6-OHDA in SNpc.

\section{Material and methods}

\subsection{Ethical approval}

Adult Wistar male rats, weighing between 200 and $250 \mathrm{~g}$ at the beginning of the protocol, were maintained in an environment with adequate temperature and ventilation $\left(22 \pm 1{ }^{\circ} \mathrm{C}\right)$ in a light-dark cycle of $12 \mathrm{~h}$. The animals had water and food ad libitum. The experiments were conducted during the light phase of the cycle. All experimental protocols are in agreement with the Brazilian Society of Laboratory Animals Science (CONCEA) and were approved by the Animal Use Ethics Committee of the State University of Londrina (process number: 19653.2016.42).

\subsection{Physical exercise}

Animals were divided into 4 experimental groups: sedentary Sham (Sham S); sedentary 6-OHDA (6-OHDA S), trained Sham (Sham T) and trained 6-OHDA (6-OHDA T). The animals that underwent exercise training (ET) followed the swimming protocol according to MartinsPinge et al. [22]. The protocol consists of 20 sessions ( 4 weeks) of $1 \mathrm{~h}$ of swimming per day, for 5 days/week. In the first week, there is an adaptation period, with a gradual increase in swimming time up to $1 \mathrm{~h}$, starting with $15 \mathrm{~min}$ on the first day, followed by $30 \mathrm{~min}$ on the second day, $45 \mathrm{~min}$ on the third and $1 \mathrm{~h}$ on the fourth day. The swimming protocol occurred during the morning in a glass tank $(100 \times 60 \times 50 \mathrm{~cm})$ containing water heated to $31 \pm 1{ }^{\circ} \mathrm{C}$ and depth of $40 \mathrm{~cm}$ (water).

\subsection{Stereotaxic surgery}

After ET or sedentarism, the animals were submitted to anesthesia with Ketamine Hydrochloride and Xylazine Hydrochloride (100 and $6.7 \mathrm{mg} / \mathrm{kg}$, ip; Ceva Santé Animale, São Paulo, Brazil) for induction of 6-OHDA Parkinsonism. They were placed in a stereotaxic device (David Kopf), and perforations in the skull of the animals were performed with a low-rotation drill, allowing the microinfusion of 6-OHDA neurotoxin $(6 \mathrm{mg} / \mathrm{mL}$ in $0.2 \%$ ascorbic acid in sterile saline solution) directly into the SNpc. For this procedure, the following stereotaxic coordinates were used as reference, from bregma: - $5.0 \mathrm{~mm}$ bregma; lateral (LL) $\pm 2.1 \mathrm{~mm}$ of the midline; dorsoventral (DV), $-8.0 \mathrm{~mm}$ of the skull [23]. The microinfusion was performed with the aid of a 30 gauge needle connected to a polyethylene tube adapted to a $10 \mu \mathrm{L}$ microsyringe (Hamilton, USA) fitted into an infusion pump. After infusion of the toxin $(3 \mathrm{~min}$ ), the needle remained in place for another $2 \mathrm{~min}$ to prevent reflux of the substance. After the surgery, the animals were kept in a temperature-controlled chamber until they recovered from the anesthesia. At the end of the surgery, all the animals received prophylactic doses $(30,000 \mathrm{IU})$ of veterinary pentabiotic (FontouraWyeth, Brazil) and were allowed 6 days for recuperation. The Sham group underwent the same surgical procedure, but with the infusion of the vehicle solution [24].

\subsection{Catheterization of femoral artery and vein}

After 6 days of the stereotaxic surgery, the animals were submitted to anesthesia with Cetamine Hydrochloride and Xilasine Hydrochloride (100 and $6.7 \mathrm{mg} / \mathrm{kg}$, ip; Ceva Santé Animale, São Paulo, Brazil) for chronic catheterization surgery of the femoral artery and vein, with the goal of monitoring the blood pressure (BP) and heart rate (HR) in the conscious state.

\subsection{Measurement of cardiovascular parameters}

After $24 \mathrm{~h}$ of catheterization, baseline cardiovascular parameters were obtained for at least $30 \mathrm{~min}$ prior to initiation of the experimental protocol. Mean arterial pressure (MAP) and HR were recorded with a pressure transducer (MLT0830) coupled to the Powerlab system4/20 T data acquisition system (ADInstruments ${ }^{\circledast}$ ). During recording, the animals were not anesthetized. After baseline recording, a $0.9 \%$ saline bolus injection $(1 \mathrm{~mL} / \mathrm{kg})$ was administered, followed after $10 \mathrm{~min}$ by an in bolus injection of L-NAME $(10 \mathrm{mg} / \mathrm{kg}$ - from Santa Cruz Biotechnology ${ }^{\circledast}$, Texas, USA), a selective inhibitor of calcium-dependent isoforms (ie, cNOS) [25,26]. After $2 \mathrm{~h}$ of recording, the animals were euthanized by decapitation and brain tissue samples were isolated. The striatum of each animal was dissected manually and weighed, thus guaranteeing homogeneity. Samples were stored at $-80^{\circ} \mathrm{C}$ until further analysis.

\subsection{Determination of striatal DA levels by high-performance liquid chromatography (HPLC)}

The striatal tissue was homogenized in $0.1 \mathrm{M}$ perchloric acid. After sonication, the homogenates were centrifuged at $13,000 \mathrm{rpm}$ for $10 \mathrm{~min}$ at $4{ }^{\circ} \mathrm{C}$ and $30 \mu \mathrm{L}$ of the supernatant were automatically injected into the chromatography system, according to Ariza and collaborators [5]. The concentrations of the substances were corrected by the mass of the tissue samples that were dissected and were expressed as nanograms of the substance per milligram of tissue. This determination confirms the efficacy of 6-OHDA injury in the SNPc, characterized by a decrease of at least $50 \%$ in striatal DA levels.

\subsection{HR and systolic arterial pressure (SAP) variability}

The 10 min recording of arterial pressure from basal were processed with the LabChart 7 Pro computer program (ADInstruments), which was able to detect the inflection points in the pulse pressure that generated a beat-by-beat time series of pulse interval (PI) and SAP. The time-frequency domain (PI and SAP variability) and power spectra were performed with a custom software (CardioSeries V2.4, http://www. danielpenteado.com). For the power spectral analysis of the PI and SAP variability, the beat-by-beat series of these parameters were resampled with data points every $100 \mathrm{~ms}$ by cubic spline interpolation $(10 \mathrm{~Hz})$. Then, the interpolated series were divided into half-overlapping segments of 512 points. The segments were visually inspected, and the data points affected by artifacts or nonstationary data were excluded from the analysis. Next, a Hanning window was used to attenuate the side effects, and spectra were calculated for all of the segments with a fast Fourier transform algorithm for the discrete time series. The spectra were integrated into low-frequency (LF; $0.2-0.75 \mathrm{~Hz}$ ) and high-frequency (HF; $0.75-3 \mathrm{~Hz}$ ) bands, and results were expressed in absolute $\left(\mathrm{ms}^{2}\right.$ or $\left.\mathrm{mm} \mathrm{Hg}^{2}\right)$ and normalized units (nu). Normalized values were obtained by calculating the percentage of LF and HF power with regard to the total power of the spectrum minus the very low frequency band 
(VLF; $<0.2 \mathrm{~Hz}$ ) power. To determine the cardiac sympathovagal balance, we calculated the ratio between the powers of the LF and HF bands (LF/HF) of the PI spectrum $[27,28]$.

\subsection{Spontaneous baroreflex analysis}

Baroreflex sensitivity (BRS) was evaluated by the sequence method with a custom software (CardioSeries V2.4, http://www. danielpenteado.com). The beat-to-beat time series of the PI and SAP values were used in the BRS analysis, these values were scanned searching for of four or more beats in which progressive increases in SAP were accompanied by progressive increases in PI or progressive decreases in SAP were accompanied by progressive reductions in PI. To detect the changes in SAP and PI, thresholds of $0 \mathrm{~mm} \mathrm{Hg}$ and $0 \mathrm{~ms}$, respectively, were used. A baroreflex sequence was used only when the correlation coefficient $(r)$ between SAP and PI was $\geq 0.8$. The BRS was determined from the slope of the linear regression between the SAP and PI of each baroreflex sequence. For this study, we evaluated baroreflex all gain, baroreflex up gain and down gain.

\subsection{Nitrite concentration measurement}

For the determination of NO concentration, different animals used in the other protocols were used. These animals were euthanized on the seventh day after stereotactic surgery and, in addition to the striatum that was removed to evaluate the efficacy of the lesion, they had the heart, aorta, right kidney, adrenal and plasma collected and stored in freezer $-80{ }^{\circ} \mathrm{C}$. The indirect concentration of the NO was determined by quantifying nitrite $\left(\mathrm{NO}^{2-}\right)$ after treatment of the sample with cadmium by Griess's colorimetric reaction [29].

\subsection{Statistical analysis}

The results were evaluated in relation to the normality of the data through the Shapiro Wilk test. For variables that presented normal distribution, parametric analysis was conducted and values are presented as mean \pm standard error of the mean (SEM). The two-hour response graphs were analyzed by two-way ANOVA for repeated measurements (two-way RMANOVA), followed by the Bonferroni post-test. For data that presented normal distribution and homogeneity of variance, ANOVA One-Way, followed by Bonferroni test was used. For nonparametric data, multiple comparisons were performed using the Kruskal-Wallis test followed by the Dunn test and values were plotted as the median (interquartile range values). The results were analyzed using the statistical program GraphPad Prism, 5th edition, and the differences were considered significant for a value of $\mathrm{p}<0.05$.

\section{Results}

\subsection{Determination of striatal DA concentrations}

Neurochemical analysis indicated that DA concentrations in both 6OHDA $S$ and 6-OHDA T groups were lower when compared to Sham $S$ and Sham $\mathrm{T}(\mathrm{p}<0.0001)$. These results indicated a decrease of the DA $>50 \%$, confirming the efficacy of the lesion in rats submitted to 6 OHDA bilateral infusion (Fig. 1).

\subsection{Concentrations of plasma and tissue nitrite}

From the indirect dosage of NO, it can be observed in Fig. 2 that there was an increased nitrite in the aorta of 6-OHDA $S$ rats in relation to Sham S, and there was a decrease in NO concentrations in 6-OHDA T rats when compared to 6-OHDA $S$ and Sham T ( $\mathrm{p}<0.05$ ).

For the heart, there was an increase in the Sham $\mathrm{T}$ group when compared to the Sham S and a reduction in NO in the 6-OHDA T group compared to the Sham $\mathrm{T}$ group $(\mathrm{p}<0.05)$. There was no significant difference between groups in plasma NO concentrations.

In relation to the kidney, we observed a reduction of NO in the animals 6-OHDA $S$ and Sham $\mathrm{T}$ in relation to the Sham $\mathrm{S}$. In adrenal, there was a lower concentration of NO in the 6-OHDA T group than 6OHDA S.

\subsection{Analysis of baseline cardiovascular parameters and after intravenous injection of saline $(1 \mathrm{mg} / \mathrm{kg})$ or L-NAME $(10 \mathrm{mg} / \mathrm{kg})$}

The baseline values for MAP, HR, systolic arterial pressure (SAP) and diastolic arterial pressure (DAP) for each group were obtained as the mean value of at least $10 \mathrm{~min}$ of baseline recording for each animal. The data showed that there was lower MAP and SAP in 6-OHDA T animals than in Sham T $(\mathrm{p}<0.05)$. Also, baseline HR was decreased in Sham $\mathrm{T}$ and 6-OHDA $\mathrm{T}$ animals relative to their controls, Sham $\mathrm{S}$ and 6OHDA S, respectively ( $\mathrm{p}<0.05$ ) (Table 1 ). Animals that underwent physical training had resting bradycardia after 4 weeks of swimming.

A bolus injection of $0.9 \%$ saline $(1 \mathrm{mg} / \mathrm{kg})$ was performed after 30 min of baseline MAP and HR, and after 10 min the bolus injection of L-NAME $(10 \mathrm{mg} / \mathrm{kg}$ ) was performed, followed of $2 \mathrm{~h}$ recording for cardiovascular parameters. The analysis was performed considering baseline values at time -20 . At time -10 , saline administration was performed and at time 0 , administration of L-NAME. The temporal evolution of the mean values of MAP and HR between the experimental groups after in bolus administration of saline and L-NAME is shown in Fig. 3.

After saline injection, there were no changes in the cardiovascular parameters of MAP and HR in all the experimental groups. Administration of L-NAME led to increased MAP and decreased HR for all animals (Fig. 3).

It can be observed in Fig. 3a that there was a lower increase in MAP at time 20 in the 6-OHDA $S$ group compared to Sham $S(p<0.05)$. At times $5,10,40,50$ and 70 there was a lower increase in the MAP of the 6-OHDA T group compared to the Sham $\mathrm{T}(\mathrm{p}<0.05$ ). Regarding HR (Fig. 3b), there was a difference between Sham $\mathrm{T}$ animals with the Sham $S$ group at times 30, 40, 80, 90 and 100 ( $p<0.05)$. Moreover, between 6-OHDA T when compared to 6-OHDA $S$ at times 50 to time 120. Trained animals maintained bradycardia for longer.

\subsection{Analysis of HR and SAP variability and spontaneous baroreflex}

Our results show a smaller variation in SAP time domain modulation of 6-OHDA $S$ animals relative to Sham $S$ ( $p<0.05$ ) (Fig. 4). In the spectral analysis of LFabs and VLFabs of SAP, there was no difference between the groups.

For the Pulse interval, there was no statistical difference in the variance in the time domain modulation. In addition, there was no difference in the LF/HF ratio and in the normalized LF and HF components (Fig. 5).

Regarding the Spontaneous Baroreflex, there was no difference between groups in the total gain; also in the "up" sequences and in the "down" sequences among the 4 groups (Fig. 6).

\section{Discussion}

The model of Parkinsonism by 6-OHDA infusion in SNpc, is a classic model, which presents behavioral and motor changes characterized of $\mathrm{PD}[24,30]$. In the present study, it can be observed that there was a decrease of $>50 \%$ in DA concentrations after 7 days of 6-OHDA infusion, which corroborates with other studies using the same model $[5,31]$. This amount of depletion has been found in animal models that attempt to mimic the early stages of PD along with the onset of motor symptoms [30,32].

Swimming for 4 weeks prior to induction of Parkinsonism did not promote neuroprotection on the decrease of DA. Data from the literature using exercise in mice prior to the lesion also did not lead to the 


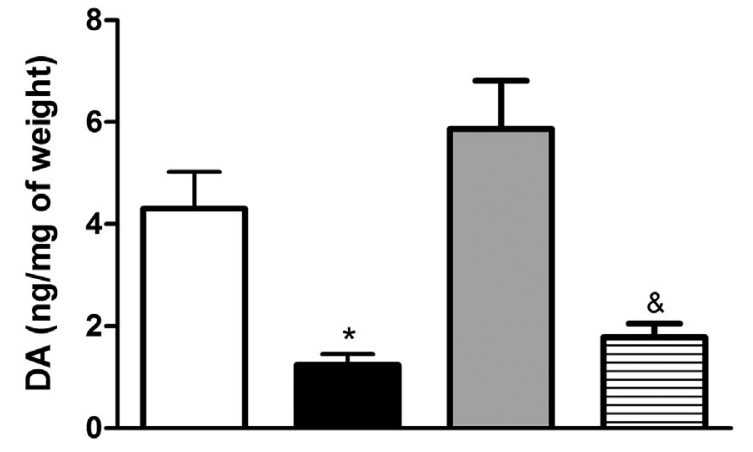

(A)

Aorta

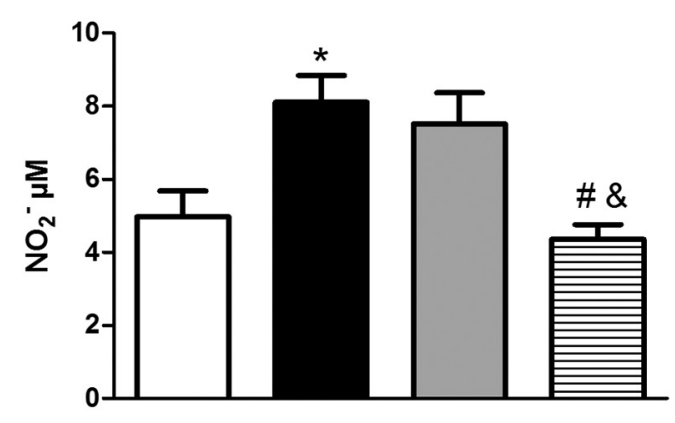

(C)

Plasma

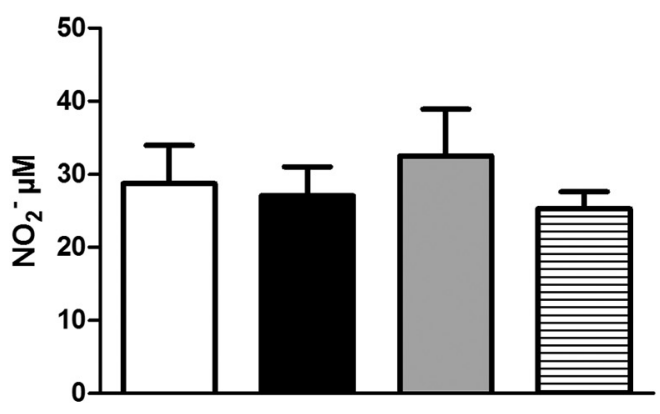

Sham S $(n=31)$

$6-O H D A S(n=30)$

Sham $T(n=23)$

6-OHDA T $(n=25)$
Fig. 1. Concentration of dopamine (DA) in the striatum quantified by HPLC after 7 days of bilateral infusion of vehicle or 6-OHDA in sedentary animals or submitted to physical training. ${ }^{*} \mathrm{p}<0.05$ vs Sham $\mathrm{S} ;{ }^{*} \mathrm{p}<0.05$ vs Sham $\mathrm{T}$. Values are expressed as mean \pm SEM. The number of groups is between parentheses (Kruskal-Wallis, followed by the Dunn test).
(B)

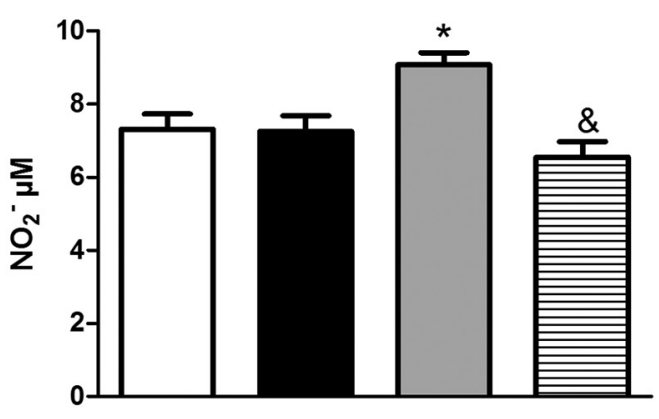

(D)

Kidney

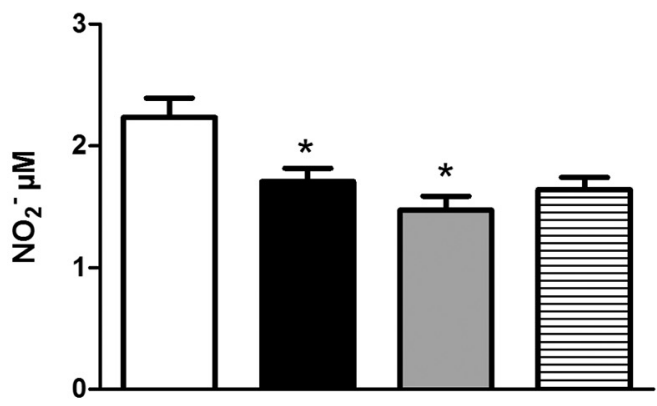

(E)

Adrenal

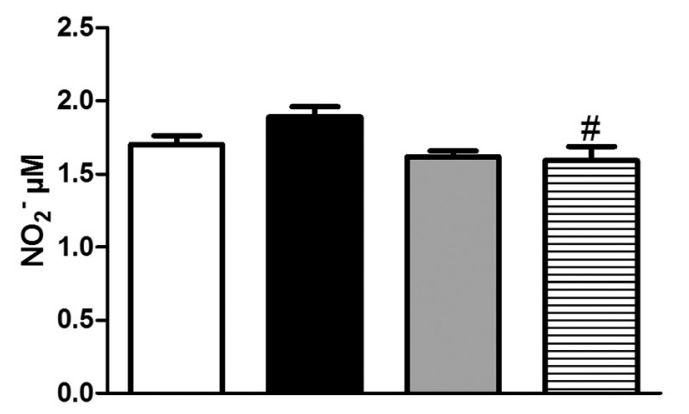

Sham S

6-OHDA S

Sham $T$

6-OHDA T

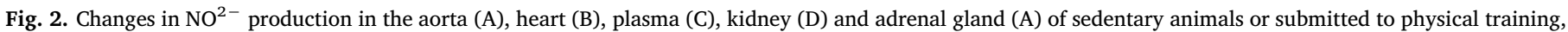

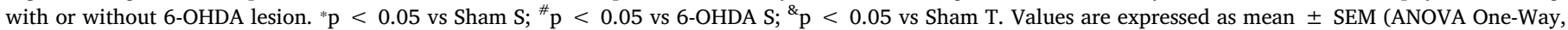

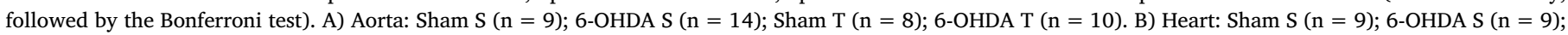

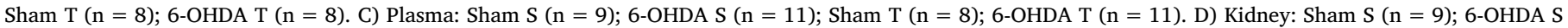
$(\mathrm{n}=9)$; Sham T $(\mathrm{n}=6)$; 6-OHDA T $(\mathrm{n}=7)$. E) Adrenal: Sham S $(\mathrm{n}=12)$; 6-OHDA S $(\mathrm{n}=10)$; Sham T (n = 7); 6-OHDA T $(\mathrm{n}=8)$. 
Table 1

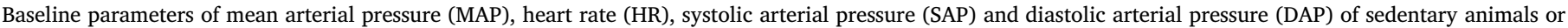
submitted to physical training, with or without 6-OHDA injury.

\begin{tabular}{|c|c|c|c|c|}
\hline & Sham S $(n=10)$ & 6-OHDA S $(n=6)$ & Sham $\mathrm{T}(\mathrm{n}=8)$ & 6-OHDA T $(\mathrm{N}=7)$ \\
\hline MAP & $106.1 \pm 1.88$ & $100.2 \pm 1.84$ & $110.1 \pm 2.38$ & $100.2 \pm 1.51^{\&}$ \\
\hline HR & $348.2 \pm 7.73$ & $355.7 \pm 4.81$ & $313.0 \pm 4.72$ & $311.9 \pm 13.53^{\#}$ \\
\hline SAP & $127.8 \pm 2.56$ & $121.3 \pm 2.08$ & $129.4 \pm 2.68$ & $118.9 \pm 1.83^{\&}$ \\
\hline DAP & $88.16 \pm 2.33$ & $82.34 \pm 2.08$ & $94.59 \pm 2.73$ & $84.97 \pm 1.79$ \\
\hline
\end{tabular}

Values are expresses as mean \pm SEM. The number of groups is between parentheses (ANOVA One-Way, followed by Bonferroni test).

* $\mathrm{p}<0,05$ vs Sham S.

\# $\mathrm{p}<0,05$ vs 6-OHDA S.

\& $\mathrm{p}<0,05$ vs Sham $\mathrm{T}$.

protection of dopaminergic neurons [33,34]. Other studies, however, showed that physical exercise before and/or after MPTP or 6-OHDA lesion promote protective and beneficial changes in these animals $[15,35,36]$. Tillerson et al. showed neuroprotection using a different model with less loss of striatal DA, in which, the 6-OHDA lesion was unilateral and led to a decrease of approximately $25 \%$ DA [37,38]. Our model induced a marked reduction of dopaminergic neurons bilaterally and in a short period of time. Gerecke and colleagues showed the importance of exercise time and intensity: in mice that performed spontaneous running for one month there was no protection; after 2 months there was partial protection and after three months, there was complete protection to the loss of DA by Parkinsonism induced by MPTP [39]. Thus, in animal experiments, appropriate time, duration and intensity may reduce the lesion induced by different toxins [40].

Most studies involving models of Parkinsonism assess brain NO concentrations. Our data showed different concentrations of NO in the aorta, heart, adrenal and kidney between groups, and seems to be the first study to evaluate NO in the cardiovascular system of animals

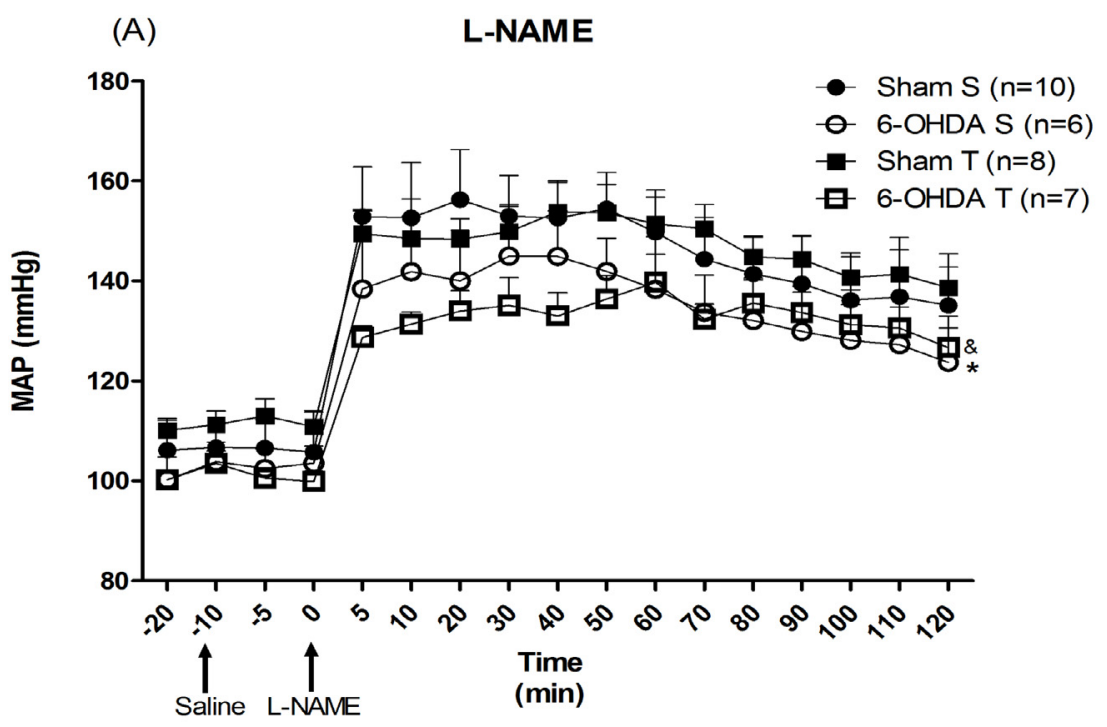

Fig. 3. Changes in time on mean arterial pressure (MAP) (A) and heart rate (HR) (B) after intravenous administration of saline (time -10) and L-NAME $(10 \mathrm{mg} / \mathrm{kg}$ - time 0$)$ in sedentary animals or submitted to physical training, with or without 6-OHDA lesion. ${ }^{*} \mathrm{p}<0.05$ vs Sham $\mathrm{S}$; ${ }^{*} \mathrm{p}<0.05$ vs 6-OHDA S; ${ }^{8} \mathrm{p}<0.05$ vs Sham T. Values are expressed as mean \pm SEM. The number of groups is between parentheses (RMANOVA Two-way, followed by the Bonferroni test). In (A), there is a significant difference between 6-OHDA $S$ and Sham $S$ at time $20(\mathrm{p}<0.05)$, and there is a significant difference between 6-OHDA T and Sham $\mathrm{T}$ at times 5,10, 40, 50 and $70(\mathrm{p}<0.05)$. In (B), there is a significant difference between Sham $T$ and Sham $S$ at times 30, 40, 80, 90 and 100 ( $\mathrm{p}<0.05$ ), and between 6-OHDA T relative to 6-OHDA $S$ at times 50 to time 120 .

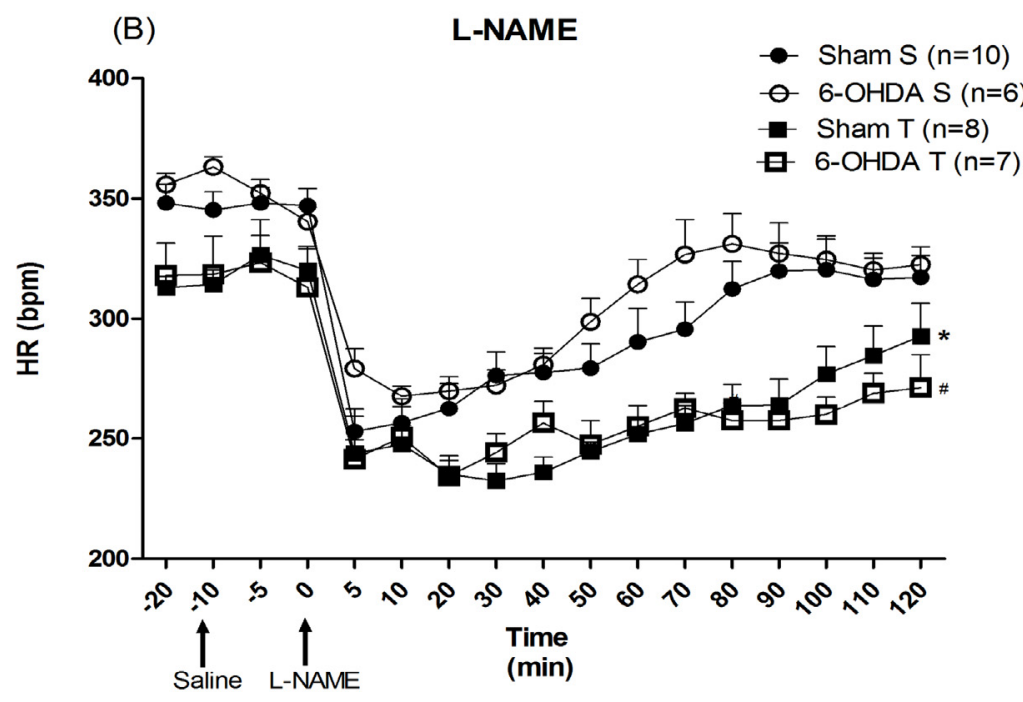



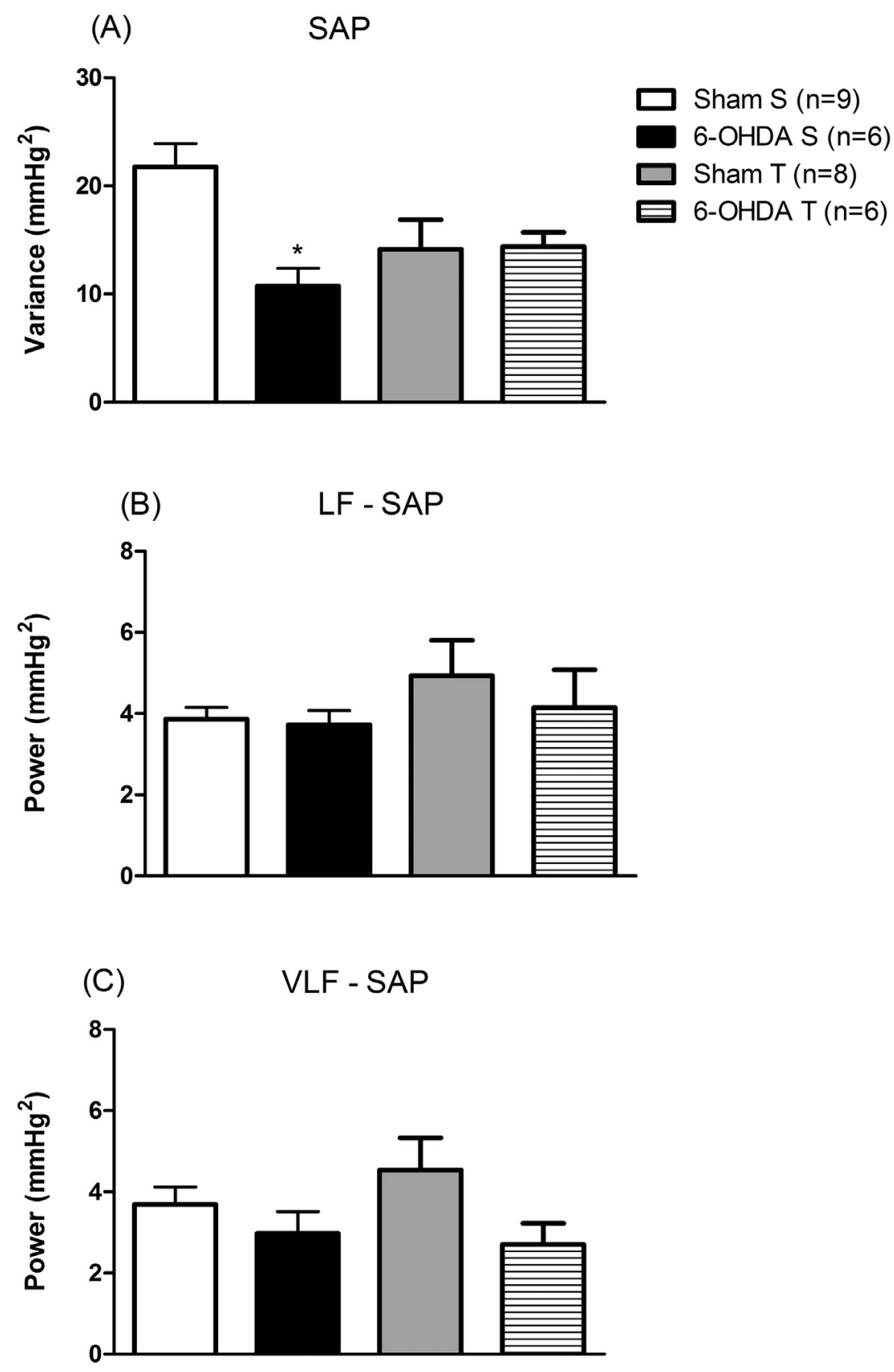

Fig. 4. Analysis of the autonomic modulation in (A) SAP variance, (B) LFabs and (C) VLFabs. Systolic arterial pressure (SAP) variability was performed in sedentary or submitted animals to physical training, with or without 6-OHDA lesion. ${ }^{*} \mathrm{p}<0.05$ vs Sham $\mathrm{S}$; ${ }^{\#} \mathrm{p}<0.05$ vs 6-OHDA S; $<<0.05$ vs Sham T. Values are expressed as mean \pm SEM. The number of groups is between parentheses (ANOVA One-way ANOVA, followed by the Bonferroni). LF, low frequency; HF, high frequency; VLF, very low frequency.

induced to Parkinsonism. A study evaluating peripherally NO in the gastrointestinal system of animals induced unilaterally by 6-OHDA lesion showed a reduction in the expression of enteric nNOS [41]. It is possible that the nitrergic system may be involved in many other aspects of Parkinson's disease.

In different studies it was shown that there is an increase in iNOS and a decrease of nNOS expression in the substantia nigra $[19,20]$. In humans, an increase in iNOS expression was found in the SNPc in brain postmortem samples from individuals with PD [21]. Considering those differences in NOS expression, it is possible that increased NO in the aorta of 6-OHDA $S$ animals may be due to increased iNOS expression. After 4 weeks of swimming, we observed an increase in NO in the heart, which may be due to increased eNOS, as regular physical activity leads to increases in eNOS gene expression and, subsequently, eNOS protein
[42]. In our study, aorta, heart and adrenal gland of 6-OHDA T animals presented a decrease in NO. In humans with heart failure, a 6-month physical training program was effective in reducing iNOS expression in skeletal muscle [43]. It is suggested that physical exercise by swimming may have promoted this same effect in the control of NO production by iNOS in the aorta and in the heart of animals induced by 6-OHDA Parkinsonism. However, additional studies should be performed to evaluate the expression of the different isoforms in these different experimental groups.

In relation to the baseline cardiovascular parameters, there were no changes in the MAP and HR values of the 6-OHDA $S$ groups in relation to Sham S, which corroborates with data from the literature using the same model [31]. The present study confirms previous data from the literature that swimming does not alter MAP values in rats, but induces 
(A)

$\mathrm{Pl}$

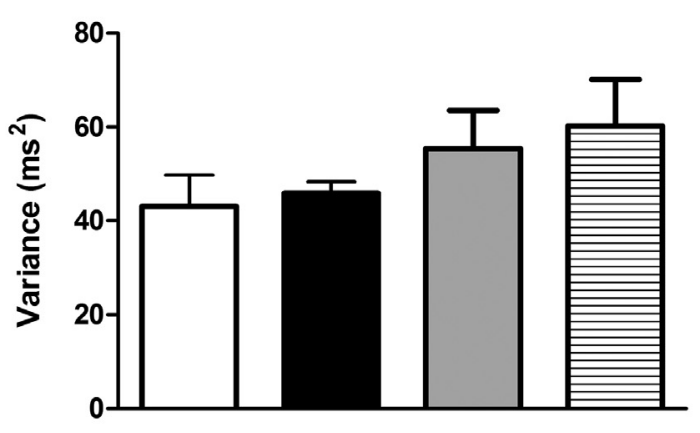

(C)

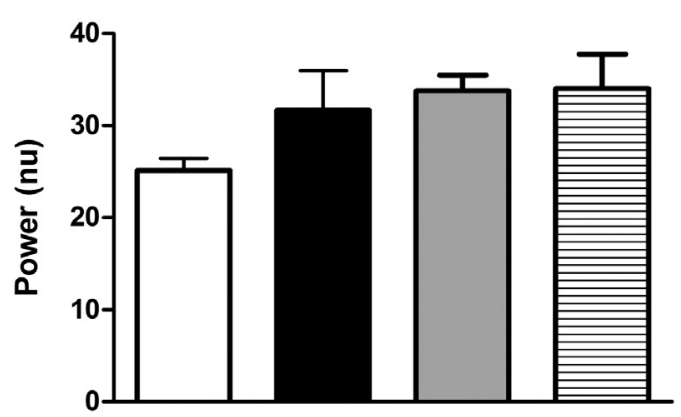

(B)

LF/HF - PI

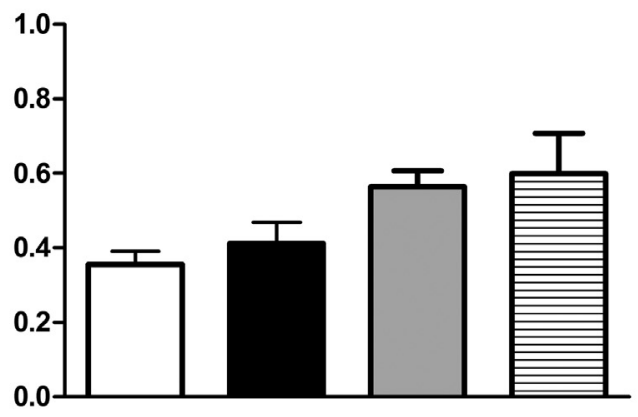

(D)

$\mathrm{HF}-\mathrm{PI}$

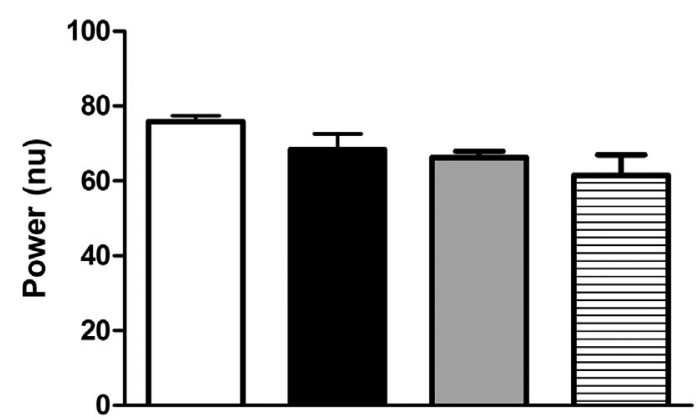

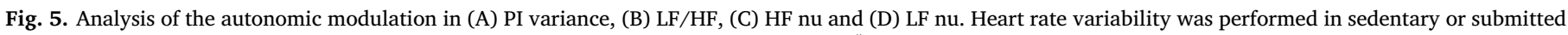

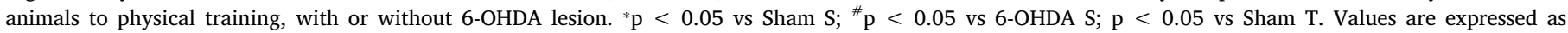

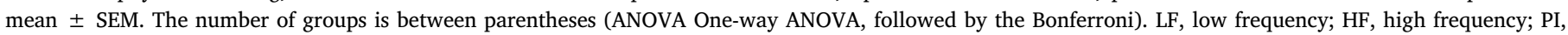
pulse interval.

resting bradycardia $[10,12,44,45]$. In addition, it shows that this bradycardia remained present even after 7 days after the last day of swimming in both the Sham $\mathrm{T}$ group and the 6-OHDA T group in relation to their controls.

Inhibition of NOS activity by L-NAME promoted hypertension and bradycardia in all groups, showing the tonic participation of NO in cardiovascular control. The dose of $10 \mathrm{mg} / \mathrm{kg}$ was chosen according to the study by Chen and $\mathrm{Hu}$ [46], which observed the maximum response of MAP increase and HR decrease with this dose [46]. L-NAME undergoes hydrolysis and forms L-NG-nitroarginine (L-NNA), which interacts non-covalently with the three isoforms of NOS. When it binds with nNOS and eNOS, the binding is slower and time dependent, while with iNOS is rapidly reversed (reviewed by Vitecek et al. [26]). Data in the literature show post-L-NAME hypertension in animals at the same dose and at the same time [25,47].

The response to L-NAME in MAP was influenced by the lesion, since in the curve there was a smaller increase of MAP after $20 \mathrm{~min}$ in the 6OHDA S group compared to Sham S. Also, at times 5, 10, 40, 50 and 70 there was a smaller increase of the MAP of the 6-OHDA T group in relation to Sham T. As previously mentioned, L-NAME seems to be more involved with inhibition of the constitutive pathway, suggesting that eNOS nitrergic tonus is lower in animals injured by 6-OHDA that in Sham animals, regardless of physical training. In the response of HR to L-NAME, the response was influenced by physical training, since there was a difference between the Sham $\mathrm{T}$ group with the Sham $S$ group at times 30, 40, 80, 90 and 100, and between 6-OHDA T in relation to 6OHDA $S$ at times 50 to 120 times. Considering that this protocol of physical exercise promotes a greater baroreflex sensitivity [12], it is possible that after 4 weeks of swimming, trained animals were more able to instantaneously correct oscillations in MAP, improving cardiovascular homeostasis and maintaining bradycardia longer than sedentary ones, while BP remains above baseline.
Regarding HRV, data from the literature shows that animals induced to Parkinsonism by 6-OHDA showed reduced HRV and higher baroreflex activity [5]. HRV is a measure that can assess the ANS modulation under physical exercise and in pathological conditions. Changes in its patterns works as an indicator of health: a healthy person usually presents higher HRV, so the reduction of HRV works as an indicator of various diseases [48]. Our results show a smaller variation in SAP time domain modulation of 6-OHDA $S$ animals relative to Sham $S$, which corroborates with data from literature. However, although we showed resting bradycardia after 4 weeks of swimming, there was no alterations in HRV and in baroreflex activity, which could be due to the fact that the analysis occurred after 8 days of the last swimming day. Also, there are non-autonomic components that influence reduction in resting heart rate, such as a decrease in the intrinsic heart rate [49].

\section{Conclusions}

In summary, our data suggest that different isoforms of NO appear to be activated between the 6-OHDA and Sham groups, and physical exercise was able to modulate the amount of NO. In addition, it was possible to observe that the alterations in the response to L-NAME in the MAP were due to the lesion and the changes in the HR were consequent of the physical exercise. These functional effects may be related to the increase of NO in the aorta in the injured animals and provided a lower pressure increase after cNOS inhibition. On the other hand, the increase of NO in the heart of the trained animals is suggestive of the baroreflex effects on the HR, which could be reflected by the increased bradycardia in these animals to the increase in pressure.

\section{Acknowledgments}

The authors are grateful for support by CAPES (1583617) 


\section{(A) Baroreflex Up Gain}

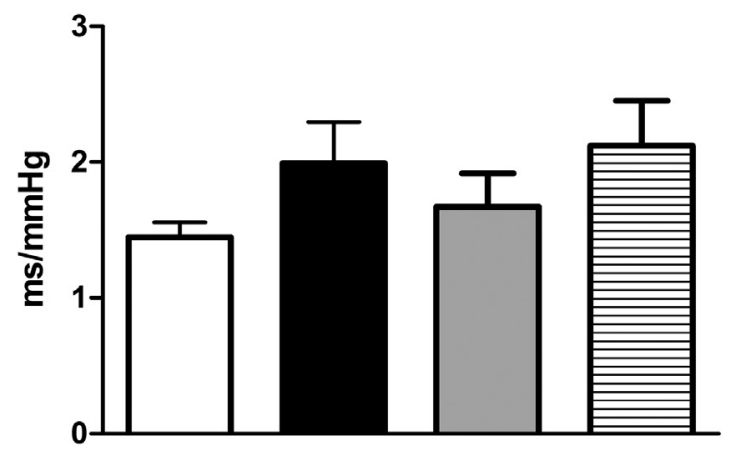

(B) Baroreflex Down Gain

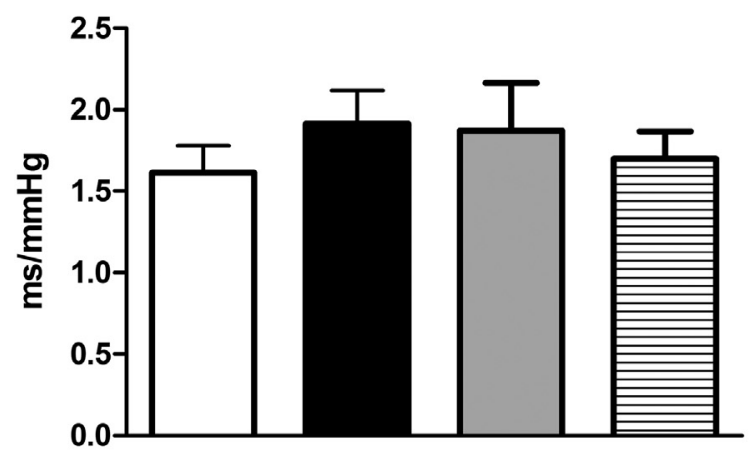

(C) Baroreflex All Gain

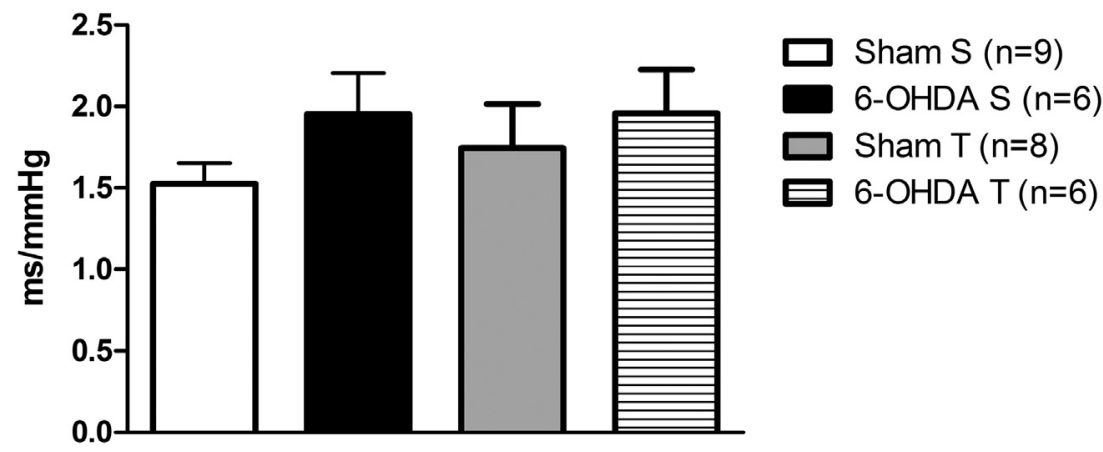

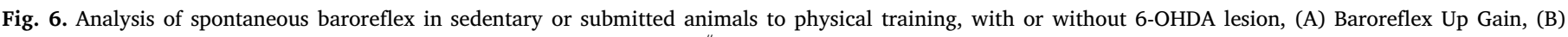

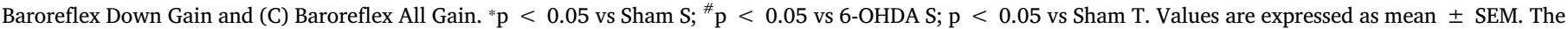
number of groups is between parentheses (ANOVA One-way ANOVA, followed by the Bonferroni test).

(Coordenação de Aperfeiçoamento de Pessoal de Nível Superior) for fellowship to L.J., CNPq (Conselho Nacional de Desenvolvimento Científico e Tecnológico), for the research fellowship to C.C.C., P.P.F., and M.C.M.P.

\section{References}

[1] F. Blandini, Neural and immune mechanisms in the pathogenesis of Parkinson's disease, J. NeuroImmune Pharmacol. 8 (1) (2013) 189-201, http://dx.doi.org/10. 1007/s11481-013-9435-y.

[2] D.J. Moore, A.B. West, V.L. Dawson, T.M. Dawson, Molecular pathophysiology of Parkinson'S disease, Annu. Rev. Neurosci. 28 (1) (2005) 57-87, http://dx.doi.org/ 10.1146/annurev.neuro.28.061604.135718.

[3] K.R. Chaudhuri, A.H. Schapira, Non-motor symptoms of Parkinson's disease: dopaminergic pathophysiology and treatment, Lancet Neurol. 8 (5) (2009) 464-474, http://dx.doi.org/10.1016/S1474-4422(09)70068-7.

[4] S. Jain, D.S. Goldstein, Cardiovascular dysautonomia in Parkinson disease: from pathophysiology to pathogenesis, Neurobiol. Dis. (2012), http://dx.doi.org/10. 1016/j.nbd.2011.10.025.

[5] D. Ariza, L. Sisdeli, C.C. Crestani, R. Fazan, M.C. Martins-Pinge, Dysautonomias in Parkinson's disease: cardiovascular changes and autonomic modulation in conscious rats after infusion of bilateral 6-OHDA in substantia nigra, Am. J. Physiol. Heart Circ. Physiol. 308 (3) (2015) H250-H257, http://dx.doi.org/10.1152/ajpheart. 00406.2014.

[6] D. Ariza, F.N.C. Lopes, C.C. Crestani, M.C. Martins-Pinge, Chemoreflex and baroreflex alterations in Parkinsonism induced by 6-OHDA in unanesthetized rats, Neurosci. Lett. 607 (2015) 77-82, http://dx.doi.org/10.1016/j.neulet.2015.09.024.

[7] B. Falquetto, M. Tuppy, S.R. Potje, T.S. Moreira, C. Antoniali, A.C. Takakura, Cardiovascular dysfunction associated with neurodegeneration in an experimental model of Parkinson's disease, Brain Res. 1657 (2017) 156-166, http://dx.doi.org/ 10.1016/j.brainres.2016.12.008.

[8] M.H. Chen, C.H. Lu, P.C. Chen, N.W. Tsai, C.C. Huang, H.L. Chen, ... W.C. Lin, Association between autonomic impairment and structural deficit in Parkinson disease, Med. (U. S.) 95 (11) (2016) 1-8, http://dx.doi.org/10.1097/MD. 0000000000003086.

[9] S.B. de Abreu, A. Lenhard, A. Mehanna, H.C.D. de Souza, F.M. de Aguiar Correa, E.M. Hasser, M.C. Martins-Pinge, Role of paraventricular nucleus in exercise training-induced autonomic modulation in conscious rats, Auton. Neurosci. Basic 
Clin. 148 (1-2) (2009) 28-35, http://dx.doi.org/10.1016/j.autneu.2009.02.007.

[10] R.B. Mastelari, H.C.D. De Souza, A. Lenhard, F.M. De Aguiar Corrêa, M.C. MartinsPinge, Nitric oxide inhibition in paraventricular nucleus on cardiovascular and autonomic modulation after exercise training in unanesthetized rats, Brain Res. 1375 (2011) 68-76, http://dx.doi.org/10.1016/j.brainres.2010.12.049.

[11] R.M. Melo, E. Martinho, L.C. Michelini, Training-induced, pressure-lowering effect in SHR: Wide effects on circulatory profile of exercised and nonexercised muscles, Hypertension, Vol. 42 2003, pp. 851-857, , http://dx.doi.org/10.1161/01.HYP. 0000086201.27420.33.

[12] H.A. Raquel, G.S. de Masson, B.F. Barna, N.G. Zanluqui, P. Pinge-Filho, L.C. Michelini, M.C. Martins-Pinge, Swimming training modulates nitric oxideglutamate interaction in the rostral ventrolateral medulla in normotensive conscious rats, Front. Physiol. 7 (June) (2016) 1-10, http://dx.doi.org/10.3389/fphys. 2016.00221.

[13] M.J. Zigmond, R.J. Smeyne, Exercise: is it a neuroprotective and if so, how does it work? Parkinsonism Relat. Disord. 20 (Suppl. 1) (2014) S123-S127, http://dx.doi. org/10.1016/S1353-8020(13)70030-0.

[14] A.T.R. Goes, L.C. Souza, C.B. Filho, L. Del Fabbro, M.G. De Gomes, S.P. Boeira C.R. Jesse, Neuroprotective effects of swimming training in a mouse model of Parkinson's disease induced by 6-hydroxydopamine, Neuroscience 256 (2014) 61-71, http://dx.doi.org/10.1016/j.neuroscience.2013.09.042.

[15] M. Al-Jarrah, M. Jamous, K. Al Zailaey, S.O. Bweir, Endurance exercise training promotes angiogenesis in the brain of chronic/progressive mouse model of Parkinson's disease, NeuroRehabilitation 26 (4) (2010) 369-373, http://dx.doi.org/ 10.3233/NRE-2010-0574.

[16] J.A. Molina, F.J. Jiménez-Jiménez, M. Ortí-Pareja, J.A. Navarro, The role of nitric oxide in neurodegeneration. Potential for pharmacological intervention, Drugs Aging 12 (4) (1998) 251-259.

[17] J.E. Yuste, M.B. Echeverry, F. Ros-Bernal, A. Gomez, C.M. Ros, C.M. Campuzano, .. M.T. Herrero, 7-Nitroindazole down-regulates dopamine/DARPP-32 signaling in neostriatal neurons in a rat model of Parkinson's disease, Neuropharmacology 63 (7) (2012) 1258-1267, http://dx.doi.org/10.1016/j.neuropharm.2012.07.031.

[18] E. Del-bel, F.E. Padovan-neto, R. Raisman-vozari, M. Lazzarini, Role of nitric oxide in motor control: implications for Parkinson's disease patho-physiology and treatment, Curr. Pharm. Des. 17 (5) (2011) 471-488, http://dx.doi.org/10.2174/ 138161211795164176.

[19] A. Czarnecka, T. Lenda, H. Domin, J. Konieczny, M. Śmiałowska, E. Lorenc-Koci, Alterations in the expression of nNOS in the substantia nigra and subthalamic nucleus of 6-OHDA-lesioned rats: the effects of chronic treatment with 1-DOPA and the nitric oxide donor, molsidomine, Brain Res. 1541 (2013) 92-105, http://dx.doi. org/10.1016/j.brainres.2013.10.011.

[20] G.T. Liberatore, V. Jackson-Lewis, S. Vukosavic, A.S. Mandir, M. Vila, W.G. Mcauliffe, ... S. Przedborski, Inducible nitric oxide synthase stimulates dopaminergic neurodegeneration in the MPTP model of Parkinson disease, Nat. Med. 5 (12) (1999) 1403-1409, http://dx.doi.org/10.1038/70978.

[21] S. Hunot, F. Boissière, B. Faucheux, B. Brugg, A. Mouatt-Prigent, Y. Agid, E.C. Hirsch, Nitric oxide synthase and neuronal vulnerability in Parkinson's disease, Neuroscience 72 (2) (1996) 355-363, http://dx.doi.org/10.1016/0306-4522(95) 00578-1.

[22] M.C. Martins-Pinge, L.K. Becker, M.R. Luccizano Garcia, D.B. Zoccal, R.V. Neto, L.S. Basso, ... O.U. Lopes, Attenuated pressor responses to amino acids in the rostral ventrolateral medulla after swimming training in conscious rats, Auton. Neurosci. Basic Clin. 122 (1-2) (2005) 21-28, http://dx.doi.org/10.1016/j.autneu.2005.07. 007.

[23] G. Paxinos, C. Watson, The Rat Brain in Stereotaxic Coordinates, Elsevier, San Diego, CA, 1998.

[24] D. Ariza, M.M.S. Lima, C.G. Moreira, P.A. Dombrowski, T.V. Avila, A. Allemand, .. M.A.B.F. Vital, Intranigral LPS administration produces dopamine, glutathione but not behavioral impairment in comparison to MPTP and 6-OHDA neurotoxin models of Parkinson's disease, Neurochem. Res. 35 (10) (2010) 1620-1627, http://dx.doi. org/10.1007/s11064-010-0222-3.

[25] J.C. Castardo-De-Paula, B.H. De Campos, E.D.T. Amorim, R.V. Da Silva, C.C. De Farias, L. Higachi, ... M.C. Martins-Pinge, Cardiovascular risk and the effect of nitric oxide synthase inhibition in female rats: the role of estrogen, Exp. Gerontol. 97 (July) (2017) 38-48, http://dx.doi.org/10.1016/j.exger.2017.07.016.

[26] J. Víteček, A. Lojek, G. Valacchi, L. Kubala, Arginine-based inhibitors of nitric oxide synthase: therapeutic potential and challenges, Mediators of Inflammation, 2012, p. 2012, , http://dx.doi.org/10.1155/2012/318087.

[27] S.G.V. Dutra, A.P.M. Pereira, G.C.S.V. Tezini, J.H. Mazon, M.C. Martins-Pinge, H.C.D. Souza, Cardiac autonomic modulation is determined by gender and is independent of aerobic physical capacity in healthy subjects, PLoS One (2013) 8, http://dx.doi.org/10.1371/journal.pone.0077092.

[28] G.C. Tezini, D.P. Dias, H.C. Souza, Aerobic physical training has little effect on cardiovascular autonomic control in aging rats subjected to early menopause, Exp Gerontol. 48 (2013) 147-153, http://dx.doi.org/10.1016/j.exger.2012.11.009.

[29] J.A. Navarro-Gonzálvez, C. García-Benayas, J. Arenas, Semiautomated measurement of nitrate in biological fluids, Clin. Chem. 44 (3) (1998) 679-681.

[30] S. Duty, P. Jenner, Animal models of Parkinson's disease: a source of novel treatments and clues to the cause of the disease, Br. J. Pharmacol. 164 (4) (2011)
1357-1391, http://dx.doi.org/10.1111/j.1476-5381.2011.01426.x.

[31] A.S. Silva, D. Ariza, D.P.M. Dias, C.C. Crestani, M.C. Martins-Pinge, Cardiovascular and autonomic alterations in rats with Parkinsonism induced by 6-OHDA and treated with L-DOPA, Life Sci. 127 (2015) 82-89, http://dx.doi.org/10.1016/j.lfs. 2015.01.032.

[32] M.C. Rodriguez-Oroz, M. Jahanshahi, P. Krack, I. Litvan, R. Macias, E. Bezard, J.A. Obeso, Initial clinical manifestations of Parkinson's disease: features and pathophysiological mechanisms, Lancet Neurol. 8 (12) (2009) 1128-1139, http://dx. doi.org/10.1016/S1474-4422(09)70293-5.

[33] A.S. Aguiar, F.S.M. Tristão, M. Amar, C. Chevarin, V. Glaser, R. De Paula Martins, ... R.D.S. Prediger, Six weeks of voluntary exercise don't protect C57BL/ 6 mice against neurotoxicity of MPTP and MPP +, Neurotox. Res. 25 (2) (2014) 147-152, http:// dx.doi.org/10.1007/s12640-013-9412-5.

[34] S.J. O'Dell, N.B. Gross, A.N. Fricks, B.D. Casiano, T.B. Nguyen, J.F. Marshall, Running wheel exercise enhances recovery from nigrostriatal dopamine injury without inducing neuroprotection, Neuroscience 144 (3) (2007) 1141-1151, http://dx.doi.org/10.1016/j.neuroscience.2006.10.042.

[35] C.J. Faherty, K. Raviie Shepherd, A. Herasimtschuk, R.J. Smeyne, Environmental enrichment in adulthood eliminates neuronal death in experimental Parkinsonism, Brain Res. Mol. Brain Res. 134 (1) (2005) 170-179, http://dx.doi.org/10.1016/j. molbrainres.2004.08.008

[36] T. Tuon, S.S. Valvassori, J. Lopes-Borges, T. Luciano, C.B. Trom, L.A. Silva, ... R.A. Pinho, Physical training exerts neuroprotective effects in the regulation of neurochemical factors in an animal model of Parkinson's disease, Neuroscience 227 (2012) 305-312, http://dx.doi.org/10.1016/j.neuroscience.2012.09.063.

[37] J.L. Tillerson, A.D. Cohen, W.M. Caudle, M.J. Zigmond, T. Schallert, G.W. Miller, Forced nonuse in unilateral parkinsonian rats exacerbates injury, J. Neurosci. 22 (15) (2002) 6790-6799, http://dx.doi.org/10.1523/JNEUROSCI.22-15-06790. 2002.

[38] J.L. Tillerson, A.D. Cohen, J. Philhower, G.W. Miller, M.J. Zigmond, T. Schallert, Forced limb-use effects on the behavioral and neurochemical effects of 6-hydroxydopamine, J. Neurosci. 21 (12) (2001) 4427-4435, http://dx.doi.org/10.1523/ JNEUROSCI.21-12-04427.2001.

[39] K.M. Gerecke, Y. Jiao, A. Pani, V. Pagala, R.J. Smeyne, Exercise protects against MPTP-induced neurotoxicity in mice, Brain Res. 1341 (2010) 72-83, http://dx.doi. org/10.1016/j.brainres.2010.01.053.

[40] L. Hou, W. Chen, X. Liu, D. Qiao, F.-M. Zhou, Exercise-induced neuroprotection of the nigrostriatal dopamine system in Parkinson's disease, Front. Aging Neurosci. 9 (November) (2017), http://dx.doi.org/10.3389/fnagi.2017.00358.

[41] F. Blandini, B. Balestra, G. Levandis, M. Cervio, R. Greco, C. Tassorelli, ... M. Tonini, Functional and neurochemical changes of the gastrointestinal tract in a rodent model of Parkinson's disease, Neurosci. Lett. 467 (3) (2009) 203-207, http://dx.doi. org/10.1016/j.neulet.2009.10.035.

[42] R.M. McAllister, M.H. Laughlin, Vascular nitric oxide: effects of physical activity, importance for health, Essays Biochem. 42 (2006) 119-131, http://dx.doi.org/10. 1042/bse0420119.

[43] S. Gielen, V. Adams, A. Linke, S. Erbs, S. Möbius-Winkler, A. Schubert, .. R. Hambrecht, Exercise training in chronic heart failure: correlation between reduced local inflammation and improved oxidative capacity in the skeletal muscle, Eur. J. Cardiovasc. Prev. Rehabil. 12 (4) (2005) 393-400, http://dx.doi.org/10. 1097/01.hjr.0000174824.94892.43.

[44] A. Medeiros, E.M. Oliveira, R. Gianolla, D.E. Casarini, C.E. Negrão, P.C. Brum, Swimming training increases cardiac vagal activity and induces cardiac hypertrophy in rats, Braz. J. Med. Biol. Res. 37 (12) (2004) 1909-1917, http://dx.doi. org /10.1590/S0100-879X2004001200018.

[45] A. Mehanna, D.C. Vitorino, C. Panis, E.E.A. Blanco, P. Pinge-Filho, M.C. Martins Pinge, Cardiovascular and pulmonary effects of NOS inhibition in endotoxemic conscious rats subjected to swimming training, Life Sci. 81 (16) (2007) 1301-1308, http://dx.doi.org/10.1016/j.lfs.2007.09.006.

[46] H.I. Chen, C.T. Hu, Endogenous nitric oxide on arterial hemodynamics: a comparison between normotensive and hypertensive rats, Am. J. Phys. 273 (1997) H1816-1823.

[47] S.M. Gardiner, Compton, a M., Kemp, P. a, \& Bennett, T., Regional and cardiac haemodynamic responses to glyceryl trinitrate, acetylcholine, bradykinin and en dothelin-1 in conscious rats: effects of NG-nitro-L-arginine methyl ester, Br. J. Pharmacol. 101 (3) (1990) 632-639, http://dx.doi.org/10.1111/j.1476-5381.1990. tb14132.x.

[48] L.C.M. Vanderlei, C.M. Pastre, R.A. Hoshi, T.D. de Carvalho, M.F. de Godoy, Basic notions of heart rate variability and its clinical applicability, Rev. Bras. Cir. Cardiovasc. 24 (2) (2009) 205-217, http://dx.doi.org/10.1590/S010276382009000200018

[49] S. Bahrainy, W.C. Levy, J.M. Busey, J.H. Caldwell, J.R. Stratton, Exercise training bradycardia is largely explained by reduced intrinsic heart rate, Int. J. Cardiol. 222 (2016) 213-216, http://dx.doi.org/10.1016/j.ijcard.2016.07.203.

[50] M.K. Barthwal, N. Srivastava, M. Dikshit, Role of nitric oxide in a progressive neurodegeneration model of Parkinson's disease in the rat, Redox Rep. 6 (5) (2001) 297-302 Disponível em: http://www.ncbi.nlm.nih.gov/entrez/query.fcgi? cmd= Retrieve\&db $=$ PubMed\&dopt $=$ Citation\&list_uids $=11778847$ 